\title{
A espiritualidade nas pessoas idosas: influência da hospitalização
}

\author{
Spirituality in the elderly: the influence of hospitalization
}

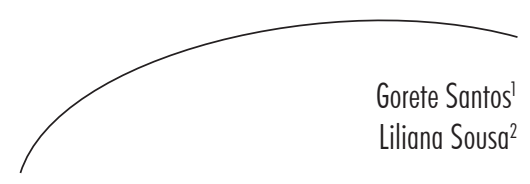

\section{Resumo}

A espiritualidade é a busca pessoal do significado e propósito da vida, e assume relevância na velhice. A hospitalização na velhice é vivida com muita ansiedade, tendo a espiritualidade demonstrado ser uma boa estratégia de coping. Este estudo procura analisar a influência de variáveis sociodemográficas, patologia e tempo de internamento. A amostra é constituída por 250 participantes ( $\geq 65$ anos), sendo 50,4\% do sexo feminino. A Escala de Espiritualidade, composta por cinco itens organizados em escala de Likert de cinco pontos, foi administrada por entrevista. Os principais resultados mostram que: a) $57 \%$ dos participantes mantêm a média de espiritualidade na admissão e alta (57\%), sendo que 22,5\% mantêm espiritualidade baixa e 22,5\% mantêm espiritualidade elevada; b) $43 \%$ dos participantes revelam alterações na espiritualidade, 21,9\% apresentam diminuição entre admissão e alta e 20,8\% demonstram aumento. Os dados indicam que os idosos que vivem em casal são os que tendem a aumentar a espiritualidade durante a internação. A hospitalização pode ter impactos diferentes na espiritualidade das pessoas idosas, por isso deve ser valorizada pelos profissionais de saúde.

\section{Abstract}

Spirituality is the personal search for meaning and purpose in life which assumes particular relevance in old age. Hospitalization in old age is a period of high anxiety, and spirituality has shown to be a relevant coping strategy. This study compares old people's spirituality at hospital admission and discharge, analysing the influence of socio-demographic variables, pathology and period of hospitalization. The sample comprises 250 participants ( $\geq 65$ years old), 50.4\% female. The Scale of Spirituality, a 5 -point Likert scale comprising five items, was administered by interview at admission and discharge. Main findings suggest that: i) 57\% of participants show similar mean of spirituality when comparing admission and discharge, $22.5 \%$ maintain low spirituality and $22.5 \%$ maintain high spirituality; ii) $43 \%$ reveal alterations in their spirituality,

Palavras-chave:

Espiritualidade.

Hospitalização.

Envelhecimento.
Key words: Spirituality. Hospitalization. Aging.

\footnotetext{
Programa Doutoral em Geriatria e Gerontologia, Departamento de Ciências da Saúde. Universidade de Aveiro. Aveiro, Portugal.

2 Secção Autónoma de Ciências da Saúde, Departamento de Ciências da Saúde. Universidade de Aveiro. Aveiro, Portugal.
}

Correspondência / Correspondence

Gorete Santos

E-mail: gorete_@hotmail.com 
$21.9 \%$ show decreasing of spirituality between admission and discharge and $20.8 \%$ show increase. Those participants living in couple tend to show increase in their spirituality. Hospitalization may have different impacts in the spirituality of elderly, what should be considered by practitioners when delivering care.

\section{INTRODUÇÃO}

A espiritualidade emerge como relevante, especialmente no processo de envelhecimento, por ser uma busca pessoal de significado para a vida. ${ }^{1}$ Além disso, a espiritualidade permite a redução/gestão da sensação de perda de controle e esperança; pode ser um recurso de coping; ajuda na aceitação da doença, segurança e otimismo face ao tratamento; e prepara para a ideia de finitude. Atualmente, existe grande interesse na pesquisa sobre a espiritualidade, sobretudo porque os dados têm demonstrado uma relação positiva e estatisticamente significativa entre espiritualidade, saúde e qualidade de vida. $^{2}$ Muitos desses estudos, contudo, focam a doença oncológica e cuidados paliativos, exigindo o alargamento a outros contextos e doenças. ${ }^{3,4}$

A definição de espiritualidade não é consensual, mas a maioria das definições refere uma procura existencial de significado e sentido para questões complexas da vida. Neste estudo, adotamos a definição de espiritualidade do National Cancer Institute (2006): 5 "sentimentos e crenças profundas, muitas vezes religiosas, incluindo a paz de espírito, conexão aos outros e as crenças sobre o significado e o propósito da vida". Esta definição parece conciliar algumas das questões conceituais em discussão, e subjaz a Escala da Espiritualidade ${ }^{4}$ utilizada neste estudo.

De fato, envolve e articula diversas conceitualizações, tais como a busca pessoal da compreensão de questões fundamentais da vida, referindo que esta relação com o sagrado ou transcendente pode ou não levar à prática de rituais religiosos. ${ }^{3,6}$ Ou seja, a espiritualidade não é um monopólio das religiões, mas uma dimensão humana, que pode ou não envolver práticas religiosas e pode existir em indivíduos ateístas ou agnósticos. ${ }^{7,8}$ Compreender a espiritualidade ultrapassa ideologias ou instituições religiosas, pois é um recurso interno do indivíduo, que pode ser acionado pelo contato com a natureza, artes ou experiência pessoal.

É necessário distinguir espiritualidade e religião, pois são conceitos com frequência utilizados de forma indiferenciada. A espiritualidade está relacionada com o sentido de vida, com o transcendente, enquanto a religião envolve também a participação ou adesão a rituais religiosos. ${ }^{9}$

Este estudo realizou-se em Portugal, onde existem poucos estudos populacionais centrados na espiritualidade e crenças religiosas. Os últimos dados oficiais sobre a religião em Portugal foram realizados pelo INE (censos populacionais, 2001) ${ }^{10}$ e indicam que: $96 \%$ da população portuguesa refere ter uma crença religiosa $(84,5 \%$ - católica; $1,41 \%$ - outra religião cristã; $0,55 \%$ - protestante; $0,20 \%$ - ortodoxa; $0,13 \%$ - muçulmanos; $0,02 \%$ - judeus); 3,9\% referem não ter qualquer religião. Estes resultados sugerem a importância da religiosidade na população portuguesa, indicando que a religião católica é predominante. ${ }^{10}$

Emníveis nacional einternacional, são escassos os estudos que focam o impacto da hospitalização de pessoas idosas na sua espiritualidade. A hospitalização, em qualquer grupo etário, é um momento difícil para a pessoa que o vivencia, e na velhice torna-se mais complexo, pois é mais facilmente associado a morte, dependência e doença, e implica grande estresse e ansiedade. Uma hospitalização reforça os sentimentos negativos da pessoa idosa, e a depressão tende a emergir ou acentuar. As pessoas idosas ficam mais frágeis e tensas durante o período de internamento e têm a sensação de isolamento. ${ }^{11}$ 
Além disso, nos meses seguintes à alta hospitalar, tendem a apresentar declínio funcional e maior morbilidade e mortalidade, segundo Ponzetto et al., ${ }^{12} \operatorname{com} 20,2 \%$ (seis meses após o primeiro internamento) de institucionalização e morte.

As doenças crônicas são um dos principais problemas das pessoas idosas. Hayes ${ }^{13}$ indica que em cada pessoa idosa independente existem três problemas crônicos, que são a principal causa de internamento hospitalar, devido à descompensação da doença. A pessoa idosa tende a apresentar taxas de internamento hospitalar mais elevadas do que as observadas noutros grupos etários, bem como maior duração do internamento. ${ }^{14}$ Alguns dados hospitalares portugueses indicam que mais de um terço das altas hospitalares corresponde a pessoas com mais de 64 anos, sendo que cerca de 53\% têm períodos de internamento superiores a 20 dias. ${ }^{15}$ Segundo Boltz \& Harrington, ${ }^{16}$ entre 2002 e 2017, haverá um aumento de $78 \%$ de internamentos hospitalares de pessoas idosas e de $16 \%$ entre os restantes grupos etários.

Em Portugal não foram encontrados estudos que relacionem hospitalização e religiosidade ou espiritualidade, mas existem algumas referências internacionais. Nomeadamente, Anandarajah \& High $^{17}$ observaram que: $94 \%$ dos doentes admitidos nos hospitais acreditam que a saúde espiritual é tão importante como a física; $77 \%$ acreditam que os médicos deveriam considerar mais as necessidades espirituais; e 37\% gostavam de falar com os médicos sobre crenças religiosas; mas $80 \%$ dos médicos nunca ou raramente discutem questões espirituais ou religiosas com os seus doentes.

Além disso, Plano Nacional de Saúde português $\left(2004 / 2010\right.$ DGS, 2004) ${ }^{18}$ indica que a dimensão espiritual deve ser contemplada no conceito de saúde para permitir a prestação holística de cuidados. Também a Carta dos Direitos dos doentes internados em Portugal ${ }^{19}$ refere que: a experiência do sofrimento torna a dimensão espiritual importante e todos os doentes têm direito a assistência religiosa sempre que o solicitarem.

\section{OBJETIVOS}

Este estudo compara a espiritualidade na pessoa idosa no momento de admissão e alta da internação hospitalar, analisando a influência de variáveis sociodemográficas (idade, estado civil, habilitações acadêmicas, local de residência, apoios sociais, patologia e tempo de internamento). Este estudo tem implicações para o desenvolvimento de medidas que visem melhorar os cuidados às pessoas idosas durante a internação hospitalar.

\section{MÉTODOS}

Foi adotada uma metodologia quantitativa, de características descritivas, comparativas e correlacionais. Este estudo foi aprovado pelo Conselho de Ética do Hospital Infante D. Pedro, E.P.E., em agosto de 2009.

Instrumentos

No estudo, utilizou-se questionário compreendendo várias questões e uma escala: dados socioeconômicos (sexo, idade, escolaridade, profissão anterior à reforma, local de residência, diagnóstico ou motivo de internamento, data de internamento no serviço, tipo de apoio social recebido); e a Escala de Avaliação da Espiritualidade. ${ }^{3}$

\section{Escala de avaliação da espiritualidade}

A escala de espiritualidade foi desenvolvida por Pinto e Pais-Ribeiro ${ }^{3}$ para avaliar a dimensão espiritual na prática clínica. Os itens foram desenvolvidos por meio da combinação da dimensão espiritual do Quality of Life-Cancer Survivor(QOL-CS), da subescala da espiritualidade do Instrument da World Health Organization Quality of Life Questionnaire (WHOQOL) e dados clínicos. ${ }^{3}$ Esta escala compreende cinco itens organizados numa escala de Likert de 5 pontos, variando entre 1 (não concordo) e 4 (plenamente de 
acordo). Pontuações mais elevadas indicam maior concordância e maior espiritualidade.

A análise fatorial realizada pelos autores da escala identificou duas subescalas: "crenças", constituída por dois itens (1- As minhas crenças espirituais dão sentido à minha vida; 2- A minha fé e crenças dão-me forças nos momentos difíceis); "esperança/otimismo" composta por três itens (3- Vejo o futuro com esperança; 4-Sinto que a minha vida mudou para melhor; 5Aprendi a dar valor às pequenas coisas da vida). ${ }^{3}$ O fator "crenças" envolve os itens associados ao sentido da vida, e o fator "esperança/otimismo" integra os itens relativos à perspectiva do futuro com esperança e redefinição de valores. $\mathrm{O}$ estudo de desenvolvimento da escala revelou boa consistência interna (Alpha de Cronbach): 0,74 para a escala global; 0,92 para o fator "crenças"; 0,69 para o fator "esperança/otimismo".

Realizamos a análise fatorial confirmatória para o nosso estudo, que revelou os mesmos dois fatores; os valores do Alpha de Cronbach neste estudo são também bons: 0,85 - escala global; 0,82 - fator "crenças"; 0,81 - fator "esperança/ otimismo".

\section{Procedimento do recolhimento de dados}

Foram aplicados 250 questionários por meio de entrevista no momento de admissão do doente no serviço, e foi aplicado o mesmo questionário no momento de alta. No momento de admissão, após o acolhimento ao doente por parte do profissional de serviço, os doentes eram contactados pela autora, que pedia sua colaboração (incluindo serem entrevistados no momento da alta). Quando os doentes tinham alta clínica, o profissional de serviço contactava a autora, que ia realizar as entrevistas. Estas ocorreram no hospital, sempre em locais que permitissem respeitar a privacidade, após a assinatura do consentimento livre e esclarecido. A coleta de dados ocorreu entre janeiro e agosto de 2010. A duração média das entrevistas foi de 30-40 minutos. Os idosos, especialmente os que vivem sós, necessitam contar suas histórias de vida e de contato social, o que tornou as entrevistas mais morosas.

Amostra

Neste estudo foram seleccionadas pessoas idosas (mais de 64 anos) internadas no serviço de Medicina 1, 2 e 3 do Hospital Infante D. Pedro, E.P.E. O local foi escolhido intencionalmente, pelo fato de a autora lá trabalhar e ser uma instituição parceira da Universidade de Aveiro.

O tamanho da amostra foi determinado após pesquisa sobre o número de doentes com idade superior a 64 anos que deram entrada no serviço de medicina interna em 2009 (ano anterior à coleta dos dados deste estudo), por meio do Gabinete de Gestão de Informação do Hospital. Verificouse que foram internadas 391 pessoas idosas no ano de 2009; uma amostra representativa desta população deveria incluir 196 participantes $^{20} \mathrm{e}$, atendendo à previsível morte experimental entre as duas aplicações (admissão e alta), optou-se por uma amostra de 250 participantes.

\section{Caracterização da amostra}

$\mathrm{Na}$ fase de admissão, a amostra é constituída por 250 participantes, $50,4 \%$ do sexo feminino (quadro 1). A média etária é de 79,63 anos (DP=7,64), residindo 59,6\% em meio urbano. Quanto ao estado civil, 49,2\% são casados, $44,4 \%$ são viúvos e $2,4 \%$ são divorciados. Observa-se que $41,6 \%$ vivem em casal, $39,2 \%$ em família e $6 \%$ estão institucionalizados. A maioria dos participantes encontra-se reformada (62\%). Em relação ao rendimento: $70 \%$ indicam ser "suficiente" e 26,4\% referem que "não chega para as necessidades". Verifica-se predomínio de baixa escolaridade, sendo a média de 2,4 anos de escolaridade $(\mathrm{DP}=2)$. Em relação ao apoio social, $85,2 \%$ estão sem apoio; $7,2 \%$ no lar de idosos; 6,8\% em apoio domiciliar. 
Quadro 1 - Caracterização da Amostra - Serviço de Medicina Interna 2010/2011. Hospital Infante D. Pedro, Aveiro, Portugal.

\begin{tabular}{|c|c|c|c|c|}
\hline \multirow{3}{*}{$\operatorname{Sexo}=\chi^{2}(1)=0,206, \mathrm{p}=0,886$} & \multicolumn{2}{|c|}{$1^{a}$ Fase (admissão) } & \multicolumn{2}{|c|}{$2^{a}$ Fase (alta) } \\
\hline & \multirow[t]{2}{*}{$\mathrm{N}$} & \multirow[t]{2}{*}{$\%$} & \multirow[t]{2}{*}{$\mathrm{N}$} & \multirow[t]{2}{*}{$\%$} \\
\hline & & & & \\
\hline Feminino & 126 & 50,4 & 93 & 51,1 \\
\hline Masculino & 124 & 49,6 & 89 & 48,9 \\
\hline \multicolumn{5}{|l|}{ Residência $=\chi^{2}(2)=0,408, \mathrm{p}=0,523$} \\
\hline Rural & 101 & 40,4 & 68 & 37,4 \\
\hline Urbana & 149 & 59,6 & 114 & 62,6 \\
\hline \multicolumn{5}{|l|}{ Estado Civil $=\chi^{2}(3)=0,475, \mathrm{p}=0,924$} \\
\hline Casado & 123 & 49,2 & 94 & 51,6 \\
\hline Divorciado & 6 & 2,4 & 3 & 1,6 \\
\hline Solteiro & 10 & 4 & 7 & 3,8 \\
\hline Viúvo & 111 & 44,4 & 78 & 42,9 \\
\hline \multicolumn{5}{|l|}{ Rendimento $=x^{2}(4)=0,489, \mathrm{p}=0,783$} \\
\hline 1. Sobra algum dinheiro & 9 & 3,6 & 6 & 3,3 \\
\hline 2. Suficiente & 175 & 70 & 133 & 73,1 \\
\hline 3. Não chega para as necessidades & 66 & 26,4 & 43 & 23,6 \\
\hline \multicolumn{5}{|l|}{ Com quem vive? ${ }^{3} \chi^{2}(5)=0,537, \mathrm{p}=0,970$} \\
\hline Casal & 104 & 41,6 & 80 & 44 \\
\hline Família & 98 & 39,2 & 68 & 37,4 \\
\hline Instituição & 14 & 5,6 & 9 & 4,9 \\
\hline Outra & 4 & 1,6 & 4 & 2,2 \\
\hline Sozinho & 30 & 12 & 21 & 11,5 \\
\hline \multicolumn{5}{|l|}{ Situação profissional $=\chi^{2}(6)=0,521, \mathrm{p}=0,914$} \\
\hline Doméstica & 2 & 0,8 & 2 & 1,1 \\
\hline Trabalhador a tempo inteiro & 10 & 4 & 8 & 4,4 \\
\hline Pensionista & 83 & 33,2 & 55 & 30,2 \\
\hline Reformado & 155 & 62 & 117 & 64,3 \\
\hline \multicolumn{5}{|l|}{ Apoio Social $=\chi^{2}(9)=13,01, \mathrm{p}=0,00$} \\
\hline $\operatorname{Sim}$ & 37 & 14,8 & 53 & 29,1 \\
\hline Não & 213 & 85,2 & 129 & 70,9 \\
\hline \multicolumn{5}{|l|}{ Se sim, qual? $=\chi^{2}(10)=4,84, \mathrm{p}=0,184$} \\
\hline Serviço de Apoio Domiciliário & 17 & 6,8 & 36 & 19,8 \\
\hline Centro de Convívio & 0 & 0 & 1 & 0,5 \\
\hline Lar de Idosos & 18 & 7,2 & 17 & 9,3 \\
\hline Acolhimento familiar & 2 & 0,8 & 1 & 0,5 \\
\hline
\end{tabular}


Em relação ao diagnóstico clínico ou motivo de internamento (categorizados de acordo com os aparelhos do corpo humano), observa-se: $36,8 \%$ com doenças respiratórias $(51,1 \%$ do sexo masculino); $18,8 \%$ com multipatologias (51,1\% do sexo feminino) e 14,4\% com problemas do aparelho neuro-hormonal $(61,1 \%$ do sexo feminino); $11,6 \%$ do aparelho circulatório $(62,06 \%$ do sexo feminino). Quanto aos antecedentes clínicos: 44\% têm mulitpatologias; 21,6\% doenças do aparelho circulatório; 9,2\% sem antecedentes clínicos relevantes (quadro 2).

Quadro 2 - Caracterização da amostra: diagnóstico e antecedentes clínicos. Serviço de Medicina Interna 2010/2011. Hospital Infante D. Pedro, Aveiro, Portugal.

\begin{tabular}{|lcccc|}
\hline \multicolumn{5}{c|}{ Diagnóstico ou motivo de internamento $=\chi^{2}(7)=0,783, \mathrm{p}=0,993$} \\
\hline Aparelho Reprodutor & 0 & 0 & 0 & 0 \\
Aparelho Circulatório & 29 & 11,6 & 22 & 8,8 \\
Aparelho Respiratório & 92 & 36,8 & 68 & 27,2 \\
Aparelho Neuro-Hormonal & 36 & 14,4 & 24 & 9,6 \\
Aparelho Digestivo & 20 & 8 & 17 & 6,8 \\
Aparelho Excretor & 18 & 7,2 & 13 & 5,2 \\
Esqueleto & 8 & 3,2 & 4 & 1,6 \\
Multipatologias & 47 & 18,8 & 36 & 14,4 \\
\hline & 23 & 9,2 & 20 & 8 \\
\hline Nenhum & 1 & 0,4 & 0 & 0 \\
Aparelho Reprodutor & 54 & 21,6 & 37 & 14,8 \\
Aparelho Circulatório & 14 & 5,6 & 10 & 4 \\
Aparelho Respiratório & 25 & 10 & 18 & 7,2 \\
Aparelho Neuro-Hormonal & 11 & 4,4 & 9 & 3,6 \\
Aparelho Digestivo & 8 & 3,2 & 7 & 2,8 \\
Aparelho Excretor & 4 & 1,6 & 3 & 1,2 \\
Esqueleto & 110 & 44 & 80 & 32 \\
Multipatologias & & & & \\
\hline
\end{tabular}

$\mathrm{Na}$ fase de alta, a amostra compreendeu 182 participantes (72,8\%); este decréscimo deveuse a: $13,2 \%(n=33)$ faleceram; 9,6\% $(n=24)$ ausentaram-se do serviço sem terem sido reinquiridos, $3,2 \%(\mathrm{n}=8)$ foram transferidos de serviço; e 1,2\% $(\mathrm{n}=3)$ recusou-se a responder. $\mathrm{O}$ tempo médio entre a $1^{\mathrm{a}}$ e a $2^{\mathrm{a}}$ inquirição foi de dez dias, variando entre três e 25 dias.
A amostra na fase de alta apresenta distribuição similar à fase de admissão (quadros 1 e 2) em todas as variáveis, com exceção da variável "apoio social", em que as distribuições são significativamente diferentes: na fase de alta, há aumento de utentes usando equipamentos sociais (serviço de apoio domiciliário e lar de idosos). Isso ocorre porque, durante a internação, 
são detectadas situações que necessitam de apoio domiciliar ou o grau de dependência dos doentes aumenta exigindo uma resposta social.

Ainda uma referência aos participantes entrevistados na admissão que morreram durante a internação: a média de idade era de 76,93 anos, 51,5\% viviam em família; 78,8\% eram aposentados; a média era de 2,4 anos de escolaridade ( $\mathrm{DP}=2,008)$. Quanto ao apoio social: $81,8 \%$ estavam sem apoio; $12,1 \% \mathrm{em}$ lar de idosos; e 6,1\% em apoio domiciliar. Em relação ao diagnóstico: $33,3 \%$ tinham doenças respiratórias; $21,2 \%$, doenças do aparelho neurohormonal; $15,2 \%$ apresentavam multipatologias. Quanto aos antecedentes clínicos: 45,5\% apresentavam multipatologias; $27,3 \%$, patologia do aparelho circulatório; e 3\% sem antecedentes clínicos relevantes.

\section{RESULTADOS}

A análise dos dados baseia-se na análise descritiva, correlacional e classificatória.

\section{Espiritualidade}

Verifica-se que as médias da escala global e subescalas (quadro 3) variam entre o "concordo um pouco" e o "concordo bastante", e são estatisticamente idênticas quando comparadas às duas fases de administração. $O$ valor mais elevado verifica-se na subescala "crenças", em ambas as fases; sendo as médias do fator "crenças" estatisticamente superiores às do fator "esperança/otimismo" (admissão: T=9,973; $p=0,04$; alta: $T=10,343 ; p=0,04)$.

Quadro 3 - Espiritualidade na fase de admissão e alta hospitalar - Serviço de Medicina Interna 2010/2011. Hospital Infante D. Pedro, Aveiro, Portugal.

\begin{tabular}{|lcccccc|}
\hline & \multicolumn{2}{c}{$1^{\mathrm{a}}$ Fase (admissão) } & \multicolumn{2}{c}{$2^{\mathrm{a}}$ Fase (alta) } & \multicolumn{3}{c|}{$\mathrm{t}$-Sudent } \\
& \multicolumn{2}{c}{$\mathrm{N}=250$} & \multicolumn{2}{c}{$\mathrm{N}=182$} & $\mathrm{t}$ & $\mathrm{P}$ \\
Crenças & $\mathrm{M}$ & $\mathrm{DP}$ & $\mathrm{M}$ & $\mathrm{DP}$ & $\mathrm{t}$ & 0.51 \\
Esperança/Otimismo & 2,76 & 0,64 & 2,72 & 0,61 & 0.654 & 0.14 \\
Escala Global & 2,35 & 0,65 & 2,26 & 0,6 & 1.467 & 0.28 \\
\hline
\end{tabular}

\section{Grupos de espiritualidade}

Para estabelecer grupos de participantes considerando a espiritualidade na admissão e alta, procedeu-se à análise de clusters (K-means) considerando todos os itens da Escala de Espiritualidade. Optou-se por uma solução de quatro clusters, por ser a mais ajustada aos resultados (quadro 4 ). 
Quadro 4 - Grupos de Espiritualidade - Serviço de Medicina Interna 2010/2011. Hospital Infante D. Pedro, Aveiro, Portugal.

\begin{tabular}{|c|c|c|c|c|c|c|c|c|}
\hline \multirow{2}{*}{$\begin{array}{l}\mathrm{N}=182 \\
\text { (Escala/Média) }\end{array}$} & \multicolumn{2}{|c|}{$\begin{array}{c}\text { Cluster } 1 \\
(\mathrm{n}=41 ; 22,5 \%) \\
\text { Manutenção de } \\
\text { pouca espiritualidade }\end{array}$} & \multicolumn{2}{|c|}{$\begin{array}{c}\text { Cluster } 2 \\
\text { (n=63; 34,6) } \\
\text { Manutenção de muita } \\
\text { espiritualidade }\end{array}$} & \multicolumn{2}{|c|}{$\begin{array}{c}\text { Cluster } 3 \\
(\mathrm{n}=40 ; 21,9 \%) \\
\text { Diminuição da } \\
\text { espiritualidade }\end{array}$} & \multicolumn{2}{|c|}{$\begin{array}{c}\text { Cluster } 4 \\
(\mathrm{n}=38 ; 20,9 \%) \\
\text { Aumento da } \\
\text { espiritualidade }\end{array}$} \\
\hline & M & DP & M & DP & M & DP & $\mathrm{M}$ & DP \\
\hline \multicolumn{9}{|c|}{ Admissão } \\
\hline Escala total $(2,51)$ & $2,04(-)$ & 0,288 & $2,93(+)$ & 0,352 & $2,95(+)$ & 0,299 & $1,83(--)$ & 0,388 \\
\hline Crença $(2,76)$ & $2,39(-)$ & 0,542 & $3,16(+)$ & 0,41 & $3,11(+)$ & 0,4 & $2,12(--)$ & 0,62 \\
\hline Esperança $(2,34)$ & $1,81(-)$ & 0,299 & $2,78(+)$ & 0,441 & $2,85(+)$ & 0,354 & $1,64(--)$ & 0,48 \\
\hline \multicolumn{9}{|c|}{ Alta } \\
\hline Escala total $(2,45)$ & $2,01(-)$ & 0,339 & $2,82(+)$ & 0,32 & $1,87(--)$ & 0,34 & $2,91(+)$ & 0,266 \\
\hline Crença $(2,72)$ & $2,34(-)$ & 0,553 & $3,10(+)$ & 0,334 & $2,10(--)$ & 0,362 & $3,17(+)$ & 0,354 \\
\hline Esperança $(2,26)$ & $1,80(-)$ & 0,364 & $2,63(+)$ & 0,454 & $1,71(-)$ & 0,441 & $2,73(+)$ & 0,318 \\
\hline
\end{tabular}

Nota: os sinais (+), (-) e (--) pretendem apenas ajudar a comparar cada média com a média global do fator.

Considerando as médias dos fatores e a evolução entre a admissão e a alta, os clusters foram nomeados assim: Cluster 1 - Manutenção de Pouca Espiritualidade, envolve participantes com baixa espiritualidade que se mantêm nas duas fases (22,5\% dos participantes); Cluster 2 - Manutenção de Muita Espiritualidade, engloba sujeitos com espiritualidade elevada que se mantêm em ambas as fases (34,6\%); Cluster 3 - Diminuição da Espiritualidade, inclui sujeitos com espiritualidade elevada na admissão, que baixa na fase de alta (21,9\%); Cluster 4 - Aumento da Espiritualidade, inclui pessoas com baixa espiritualidade na admissão e que aumenta na alta $(20,8 \%)$.

Em seguida analisou-se, com base no cálculo em frequências observadas e esperadas, como os clusters variam com o sexo, residência, estado civil, com quem vive, situação profissional, o número de visitas, diagnóstico e antecedentes clínicos. Os resultados indicam distribuições similares nas variáveis "sexo", "residência", "visitas" e "diagnóstico". Emergiram distribuições com diferenças significativas quanto a "com quem vive". Verifica-se uma tendência para que quem vive em casal vivenciar um aumento da espiritualidade; quem não vive em casal, mesmo que viva em família, tende a diminuir a espiritualidade ou manter muita espiritualidade.

Em relação a estado civil, situação profissional e antecedentes clínicos, há tendências que passamos a analisar. Quanto ao estado civil, observa-se que: no grupo dos que mantêm muita espiritualidade (cluster 2) há menos casados e mais viúvos; no cluster 4, aumento da espiritualidade, há mais pessoas casadas e menos viúvas do que esperado. Quanto à situação profissional, observa-se que (quadro 4): no cluster 3 , diminuição da espiritualidade, há mais aposentados do que esperado; no cluster 4, aumento da espiritualidade, há menos aposentados do que esperado. Quanto aos antecedentes clínicos, observa-se: no cluster 1 (manutenção de pouca espiritualidade) e no cluster 4 (aumento da espiritualidade), há mais pessoas com multipatologia do que o esperado; nos clusters 2 (manutenção de muita espiritualidade) e 3 (diminuição da espiritualidade), há menos pessoas com multipatologia do que o esperado.

Em relação às variáveis "rendimento", "escolaridade", "idade" e "dias de internação", calcularam-se as médias em cada cluster, não tendo sido encontrada qualquer diferença estatisticamente significativa. 


\section{DISCUSSÃO}

\section{Espiritualidade}

No momento de admissão hospitalar, verificou-se que os participantes atribuíram importância de moderada a alta à espiritualidade. A literatura indica que a espiritualidade ganha relevo com o envelhecimento, pois é uma estratégia que ajuda a enfrentar as alterações. ${ }^{21}$ Além disso, é um processo que responde às necessidades de desenvolvimento nas pessoas idosas, que enfrentam o processo de revisão e integração da vida, procurando um sentido para a vida que viveram e para a que vão viver. ${ }^{22}$

Neste estudo, os participantes vivem um momento de internação hospitalar, que nos mais velhos tende a ser vivido com especial vulnerabilidade, envolvendo a incerteza quanto ao futuro e, em especial, o confronto com a morte e o sentido da vida. ${ }^{11} \mathrm{O}$ coping religioso/ espiritual apresenta-se como uma forma das pessoas utilizarem a fé e crença para lidarem com a crise, permitindo às pessoas idosas, doentes e internadas procurarem um significado ou explicação para o adoecimento e/ou a cura através da fé. ${ }^{23}$

Os dados indicam que dimensão vertical (crenças) é estatisticamente superior à dimensão horizontal (esperança/otimismo). Este resultado sugere que a crença não é acompanhada por esperança e otimismo; aliás, a média da espiritualidade diminui porque no fator "esperança/otimismo" os participantes pontuam mais baixo. A dimensão "esperança/otimismo" apresenta a média mais baixa no item "vejo o futuro com esperança". Provavelmente, também pela idade, os participantes se confrontam com a percepção de finitude e não veem o futuro com esperança. ${ }^{9}$ A percepção do tempo varia com a idade (tempo de vida esperado), nos mais velhos tem de ocorrer um ajustamento que reflita a aceitação dos constrangimentos do tempo de vida possível ou provável. A teoria da seletividade sócio-emocional ${ }^{24,25}$ sugere que a forma como os indivíduos percebem o tempo se associa ao ajustamento social e emocional: i) quem percebe o tempo futuro como limitado tende a dar prioridade a objetivos emocionais; ii) os que percebem o futuro como aberto e o presente como orientado para um futuro alargado tendem a atribuir prioridade a objetivos instrumentais. As pessoas mais velhas também têm de reajustar sua percepção do tempo e definir seus objetivos em consonância.

\section{Da admissão à alta hospitalar}

As médias no momento de admissão e alta hospitalar são estatisticamente similares. Este resultado sugere que a internação hospitalar parece não alterar a espiritualidade das pessoas idosas. Contudo, para melhor compreender a evolução da espiritualidade entre o momento de admissão e alta hospitalar, realizou-se uma análise de clusters, que fez emergir quatro grupos estatisticamente diferentes. Essa análise mais discriminatória sugere diversos comportamentos dos participantes. Os quatro grupos emergentes revelam duas situações: i) dois grupos não apresentam alterações na espiritualidade entre a admissão e a alta hospitalar (57\%); num grupo há manutenção de pouca espiritualidade $(22,5 \%)$, e no outro, de muita espiritualidade $(34,6 \%)$; ii) dois grupos apresentam alterações (43\%); num há diminuição $(21,9 \%)$, e no outro, aumento da espiritualidade $(20,9 \%)$. Ou seja, a internação hospitalar envolve diferentes impactos na espiritualidade, revelando a heterogeneidade das pessoas idosas e, provavelmente, diferentes processos desenvolvimentais.

Algumas variáveis estudadas não demonstraram influenciar a evolução da espiritualidade entre a admissão e a alta hospitalar: situação profissional; diagnóstico/ motivo de internação; antecedentes clínicos; rendimento; escolaridade. Outras, entanto, ajudaram a analisar esses diferentes percursos relativos à espiritualidade durante a internação.

A variável "com quem vive" é a única estatisticamente significativa. $O$ significado de viver em família não fica completamente esclarecido pela resposta ao instrumento utilizado, pois pode incluir viver com o cônjuge 
e outros familiares, ou pode indicar que vive apenas com outros familiares porque é viúvo, solteiro ou divorciado/separado (um próximo estudo deverá clarificar esta informação).

A influência de viver em família pode assumir dois sentidos: i) manter muita espiritualidade; ii) diminuir a espiritualidade. Viver com a família na velhice tem sido associado às pessoas idosas sentirem que são uma sobrecarga para os familiares e/ou sentirem que existe uma troca desequilibrada (recebem mais do que dão). A internação hospitalar pode criar o sentimento de que a dependência e a necessidade de cuidados aumentarão e a pessoa idosa poderá sentir que a qualidade da relação familiar poderá não ser a melhor para esta situação (diminui espiritualidade); ou a pessoa idosa sente que a família se manterá dedicada e feliz por cuidar (aumento da espiritualidade).

O aumento da espiritualidade é mais observado em pessoas que vivem em casal. Diversos estudos demonstram que os idosos que vivem em casal tendem a sofrer menos de depressão, devido ao suporte conjugal que influencia o bem-estar psicológico e funciona como alívio do estresse cotidiano. ${ }^{26} \mathrm{~A}$ rede familiar pode providenciar recursos emocionais e psicológicos que permitam à pessoa evitar certos estressores; contudo, é o apoio conjugal que mais parece permitir a expressão de medos, angústias e ansiedades. Na relação conjugal, há uma relação de igualdade e complementaridade, desenvolvida e fortalecida ao longo de anos, que facilita a confiança em ter o apoio adequado.
Implicações, limites e perspectivas de pesquisa

Os resultados dão algumas indicações para os cuidados espirituais durante a internação hospitalar de pessoas idosas; mostram o relevo do agregado familiar associado ao estado civil, pois homens viúvos parecem ter mais dificuldade em manter a espiritualidade como uma estratégia de coping. Como perspectivas de investigação, consideramos relevante perceber melhor a qualidade e influência da relação conjugal e da relação familiar ao longo da vida, para aprofundar sua influência na espiritualidade e na vivência de uma internação hospitalar. A análise mais aprofundada do agregado familiar é de relevar. Este estudo é quantitativo e seria interessante complementar com dados qualitativos, para se perceber melhor o significado da espiritualidade para os participantes, como é vivida e agida durante a internação.

\section{CONCLUSÃO}

Este estudo focou a influência da hospitalização na espiritualidade da pessoa idosa. Revelou que a internação hospitalar, mesmo com duração reduzida (cerca de dez dias), influencia as vivências espirituais do idoso internado, sendo que a existência de uma relação conjugal influencia a manutenção ou o aumento da espiritualidade. Assim, é relevante que os profissionais de saúde valorizem esta dimensão, para promover o bem-estar e a qualidade de vida das pessoas idosas internadas.

4. Pinto C, Ribeiro JP. Avaliação da espiritualidade dos sobreviventes de cancro. Implicações a qualidade de vida. Revista Portuguesa de Saúde Pública. 2010; 28(1).

5. National Cancer Institute. Spirituality. 2006. [acesso em 10 de Novembro 2010]. Disponivel em: http:// www.cancer.gov/Templates/db_alpha?CrlD $=441265$

3. Pinto C, Ribeiro JP. Construção de uma escala de avaliação em contextos de saúde. Arquivos de Medicina. 2007; 21(3), 47-53.
6. Crowther MR, Parker MW, Achenbaum WA, Larimore, WL e Koenig, HG. Rowe and Kahn's 
model of successful aging revisited: positive spirituality - the forgotten factor. The Gerontologist. 2002; 42(5), 613-20.

7. Fleck MA e coutros. A avaliação de qualidade de vida-guia para profissionais de saúde. Porto Alegre: Artmed.

8. Pestana JP, Estevens D e Conboy J. O papel da espiritualidade na qualidade de vida do doente oncológico em quimioterapia. Revista de ConsCiências. 2007, Setembro.

9. Dalby P. Is there a process of spiritual change or development with ageing? A critical review of research. Aging \& Mental Health. 2006; 10(1), 4-12.

10. Instituto Nacional de Estatística. Censos 2001: resultados definitivos. Lisboa; 2002.

11. Carvalhais M e Sousa L. Comportamentos dos enfermeiros e impacto em doentes idosos em situação de internamento hospitalar. Revista Electrónica de Enfermagem. 2007; 9(3), 596-616.

12. Ponzetto M, Zanocchi M, Maero, B, Giona E, Franscisetti F, Nicola E, Fabri F. Post-hospitalization mortality in the elderly. Archives of gerontology and geriatrics. 2003; 36, 83-91.

13. Hayes KS. Idosos. In: Oman K et al. (Org.). Segredos em Enfermagem de Urgência. Porto Alegra: Artmed. p.297-304.

14. Giacomini T, Wanderley K. Compreendendo o idoso e a sua vivência de internação hospitalar. Revista Kairós Gerontologia. 2010; 13(1), 221-30.

15. Campos AC. Reformas da saúde: fio condutor. Coimbra: Almedina; 2008.

16. Boltz M, Harrington C. Nurses Improving Care for Health System Elders (NICHE). The American Journal of Nursing 2005; 105(5):101-02.
17. Anandarajah G, High E. Spirituality and Medical Practice: Using the HOPE Questions as a Practical Tool for Spiritual Assessment. American Family Physician. 2001; 63, 81-9.

18. Brasil. Ministério da Saúde. Direcção Geral de Saúde. Plano nacional de Saúde 2004-2010: mais saúde para todos. Lisboa: Direção-Geral de saúde; 2004.

19. Brasil. Ministério da Saúde. Direção Geral de Saúde. Carta dos direitos do doente internado. 2005 [acesso em 15 de Fevereiro de 2011]. Disponível em: http://www.dgs.pt/upload/membro.id/ficheiros/ i006779.pdf

20. Krejcie R, Morgan D. Determining samples size for research activities. Educational and Psychological Measurement. 1970; 30, 607-10.

21. Panzini RG, Bandeira DR, Fleck MA. Espiritualidade/Religiosidade e Qualidade de Vida. In: A avaliação da Qualidade de vida: guia para profissionais. Porto Alegre: Artmed; 2008. p.178-96.

22. Ross L. The spiritual dimension: its importance to patients health, well-being and quality of life and its implications for nursing practice. International Journal of Nurse Studies. 1995; 32, 457-68.

23. Tornstam L. Gerotranscendence: The Contemplative Dimension of Aging. Journal of Aging Studies. 1997; 11(2), 143-54.

24. Carstensen LL, Isaacowitz D, Charles ST. Taking time seriously: A theory of socioemotional selectivity. American Psychologist. 1999; 54, 165-81.

25. Lang FR, Carstensen LL. Time counts: Future time perspective, goals and social relationships. Psychology and Aging. 2002; 17, 125-39.

26. Ramos MP. Apoio social e saúde entre idosos. Sociologias. 2002; 4(7), 156-75. 